\title{
Hepatectomía laparoscópica*
}

\author{
Drs. NICOLÁS JARUFE C. ${ }^{1}$, ANDRÉS MARAMBIO G. ${ }^{1}$, Int. JUAN FRANCISCO DE LA LLERA K. ${ }^{2}$, \\ Drs. JULIÁN VARAS C. ${ }^{1}$, MARCEL SANHUEZA G. ${ }^{1}$, JORGE MARTÍNEZ C. ${ }^{1}$
}

1 Departamento de Cirugía Digestiva, División de Cirugía, Pontificia Universidad Católica de Chile.

2 Interno de Medicina. Facultad de Medicina, Pontificia Universidad Católica de Chile.

Santiago, Chile.

\begin{abstract}
\section{Laparoscopic hepatectomy}

The surgical technique employed in laparoscopic liver resection is characterized by its great complexity. It is also associated to a long learning curve, which determines that this procedure is only completed in certain reference centers by few highly trained surgeons. Other challenges related to this minimally invasive approach are greater operatory risk due to vascular control, necessity in employing highly sophisticated surgical technology and to have this expensive equipment. Though it involves stages common to other laparoscopic interventions in abdominal surgery, it also requires procedures-specific steps and knowledge such as the use of intraoperative laparoscopic ultrasound, indispensable when planning the surgical strategy and in fulfilling hepatic transection. Laparoscopic liver resection was initially used only in resecting small peripheral hepatic lesions. With the development of this technique, more complex operations have been completed such as segmentectomies and major hepatectomies. Now has even grown to develop in liver transplantation, especially in living donor cases. However, there is still controversy in employing this difficult technique in all resections, specifically in challenging procedures such as caudate or posterior segments excision. Through this article, the surgical technique employed in laparoscopic liver resection will be displayed, including pediatric living donor surgery.
\end{abstract}

Key words: Hepatectomy, laparoscopy, surgical technique.

\section{Resumen}

La técnica quirúrgica de la resección hepática laparoscópica se caracteriza por tener una complejidad importante determinando una curva de aprendizaje prolongada, lo que implica que se concentre en centros de referencia donde sólo algunos cirujanos capacitados puedan realizarlas. Además presenta un riesgo quirúrgico mayor, dado principalmente por la hemorragia, la necesidad de dispositivos quirúrgicos especiales y la posibilidad de contar con equipos laparoscópicos de óptima calidad. Si bien tiene secuencias similares a todas las técnicas laparoscópicas en cirugía abdominal, posee algunos pasos únicos tales como el uso de la ecografía intraoperatoria laparoscópica, instrumento imprescindible a la hora de planificar el abordaje a usar, y la transección hepática. Inicialmente sólo se aplicaba para resecar lesiones pequeñas y periféricas, sin embargo, con el desarrollo de la técnica se han descrito distintos tipos de resecciones, incluyendo hepatectomías mayores. También se ha desarrollado en el ámbito del trasplante, principalmente en lo que respecta a donante vivo. En este artículo se expone la técnica quirúrgica que usamos en las principales hepatectomías laparoscópicas, de segmentos laterales y hepatectomías derecha e izquierda, además de describir la técnica usada en nuestros casos de donante vivo pediátrico.

Palabras clave: Hepatectomías, laparoscopia, técnicas quirúrgicas.

*Recibido el 1 de abril de 2013 y aceptado para publicación el 12 de junio de 2013.

Los autores no refieren conflictos de interés.

Correspondencia: Dr. Nicolás Jarufe C.

Marcoleta 365, Santiago, Chile. Código Postal: 8330024. Fax: 56-2-26382793.

njarufe@med.puc.cl 


\section{Introducción}

La técnica quirúrgica en hepatectomías laparoscópicas sigue los principios básicos de la cirugía laparoscópica tradicional, en lo que respecta a la posición del paciente y los cirujanos, uso de neumoperitoneo, inserción de trócares, uso de instrumentos laparoscópicos ad-hoc y la extracción de la pieza operatoria. Todo esto irá variando según el centro, características de la lesión y disponibilidad del instrumental adecuado ${ }^{1}$. Un paso importante a agregar a esta secuencia corresponde a la transección del parénquima hepático, momento crítico en el cual existen los mayores riesgos de sangrado intraoperatorio, embolias aéreas y ocurrencia de fístulas posteriores $^{2}$. Un pilar fundamental y transversal a todos los cirujanos y patologías (especialmente las malignas) es el uso de la ecografía intraoperatoria laparoscópica (EIOL). Esta es esencial a la hora de planificar el abordaje de la lesión en varios aspectos, partiendo por la adecuada visualización de los bordes en resecciones no anatómicas y anatómicas para así lograr el margen adecuado, etapificarla correctamente al descartar la presencia de lesiones satélites no pesquisadas en las imágenes preoperatorias y como guía imprescindible a la hora de planificar la transección del parénquima ayudando en la identificación de las estructuras importantes a respetar o seccionar, tales como las venas suprahepáticas y los pedículos portales más importantes ${ }^{3}$. La EIOL pasa a ser la extensión de las manos del cirujano y viene a reemplazar la necesidad de la palpación del hígado, por lo cual debe estar siempre disponible a la hora de realizar una resección hepática laparoscópica (RHL). Un segundo aspecto a considerar es la posibilidad de controlar anticipadamente cualquier sangrado, ya sea por la transección realizada o por cualquier lesión vascular producida durante la cirugía. Al evaluar la técnica propiamente tal y compararla con cirugía abierta, un ítem frecuentemente analizado es la necesidad de realizar la maniobra de Pringle y el tiempo que esta se usa, lo que permitiría tener mejor control del sangrado parenquimatoso. Esta maniobra debe ser usada de manera selectiva ${ }^{4}$.

\section{Técnica general}

Los aspectos generales a considerar para cualquier tipo de resección hepática laparoscópica son:

\section{a. Posición del paciente}

Posición de Lloyd-Davies con el cirujano entre las piernas, aunque varía según el centro y tipo de resección hepática, pudiendo el paciente estar en decúbito supino y el cirujano a la derecha (Figura 1). Decúbito lateral izquierdo en segmentectomías VI y VII con el cirujano al lado izquierdo.

\section{b. Neumoperitoneo de $\mathrm{CO}_{2}$}

Uso de neumoperitoneo doble a través de dos insufladores e incluso aumentar la presión de $\mathrm{CO}_{2}$ a $18 \mathrm{mmHg}$, lo que permitiría mejorar el control del sangrado venoso. Además la doble insuflación permite que durante el control del sangrado se aspire en forma continua sin perder visualización por caída del neumoperitoneo a la vez de mantener una salida adecuada del humo acumulado al mantener algún puerto abierto.

\section{c. Equipo}

La óptica debe ser de $30^{\circ}$. En el caso que se vaya a resecar segmentos póstero superiores se recomienda contar con un equipo laparoscópico flexible, dado la mayor dificultad técnica, con el fin de lograr una mejor visualización de estos.

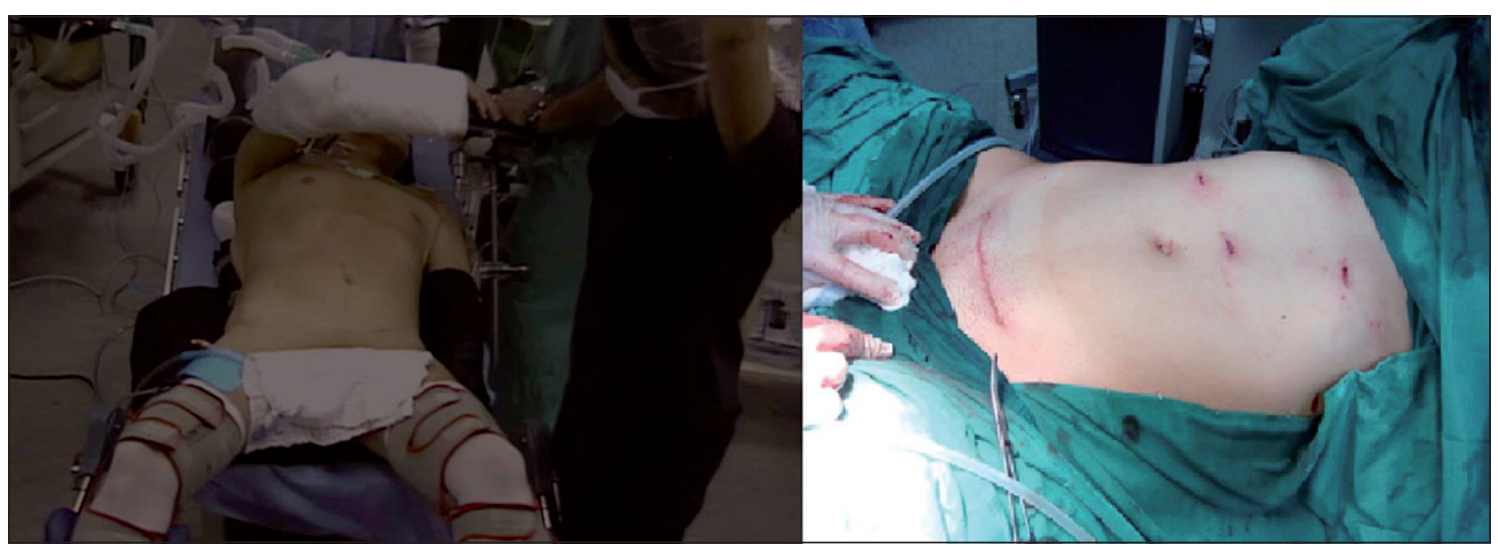

Figura 1. Posición de Lloyd-Davies. El cirujano se ubica entre las piernas del paciente. A la derecha se observa el resultado final luego del retiro de los trócares. 


\section{d. Exploración}

Lo primero es explorar toda la superficie hepática en busca de lesiones no pesquisadas y posteriormente el resto de la cavidad abdominal. Una vez descartada enfermedad diseminada se complementa la exploración con la EIOL (Figura 2).

\section{e. Instalación y ubicación de los trócares}

Habitualmente se usan 5 trócares para las hepatectomías mayores y 4 en la segmentectomía lateral o de segmentos VI y VII.

\section{f. Control vascular (maniobra de Pringle)}

Es recomendable disecar siempre el pedículo hepático y pasar una cinta alrededor de éste a fin de tener el control vascular en caso de algún accidente. Se recomienda usar esta maniobra en forma selectiva (Figura 2).

\section{g. Demarcar la transección}

una vez realizada la EIOL y teniendo claro la ubicación y los márgenes de la lesión, así como la ubicación de las principales estructuras vasculares adyacentes, es recomendable demarcar la línea de transección a seguir, lo cual se puede realizar con distintos instrumentos, como por ejemplo el hook o el coagulador de microondas (Figura 3).

\section{h. Transección y hemostasia}

Durante esta etapa es crítico contar con presiones venosas centrales bajas $\left(<3-6 \mathrm{~cm} \mathrm{H}_{2} \mathrm{O}\right)$ a fin de dismi- nuir el retorno y el sangrado hepático, lo que se puede asociar a clampeo intermitente del pedículo. En este momento vamos seccionando el parénquima a través de nuestra línea de transección, realizando hemostasia en forma paralela y controlando las estructuras que vayamos seccionando, ya sea con ligaduras, clips o stapler. Se han propuesto varias técnicas de transección, según la preferencia del cirujano, no existiendo superioridad demostrada de un método sobre otro. De esta forma existen diversos instrumentos con distintos mecanismos de funcionamiento, capaces de seccionar el hígado y luego permitir el control de la hemostasia ${ }^{5}$.

\section{a. Transección}

Por un lado contamos con los disectores ultrasónicos, los cuales pueden ser de bajo o alto poder. Los de bajo poder fraccionan los tejidos que contienen agua dejando indemnes las estructuras que contienen poca cantidad de agua, como los vasos sanguíneos o conductos biliares. Un ejemplo de este tipo de disector lo constituye el CUSA ${ }^{\mathrm{TM}}$ (Cavitron Ultrasonic Surgical Aspirator) el cual fragmenta el tejido a través de la energía ultrasónica (su punta vibra a una frecuencia de $23 \mathrm{kHz}$ ) y luego lo aspira, exponiendo las estructuras vasculares y biliares que luego pueden ser ligadas o controladas con clips. Los de alto poder fraccionan los tejidos por calor y de esta forma cortan y coagulan los bordes al mismo tiempo. Un ejemplo de este tipo lo constituye el bisturí harmonico ${ }^{\mathrm{TM}}$ (Figura 3 y 4) y el Sonicision ${ }^{\mathrm{TM}}$, los cuales cortan y coagulan el tejido


Figura 2. Usando la ecografía intraoperatoria laparoscópica (EIOL). A la derecha se observa la maniobra de Pringle.

Figura 3. Demarcando el área de transección con el apoyo de EIOL. A la derecha se observa la transección del parénquima. 
mediante un mecanismo de vibración de una de sus hojas, la cual oscila a una frecuencia de $55,5 \mathrm{kHz}$, no superando nunca los $100^{\circ} \mathrm{C}$, causando mínimo daño a los tejidos adyacentes. Otros instrumentos de sección de uso habitual corresponden al LigaSure ${ }^{\mathrm{TM}}$ y EnSeal ${ }^{\mathrm{TM}}$, los cuales combinan compresión y energía bipolar, fusionando el colágeno y elastina de los vasos, formando un sello permanente que le permite ligar vasos de hasta $7 \mathrm{~mm}$ (el bisturí harmonico sólo sella vasos de hasta $3 \mathrm{~mm}$ ). Con menor popularidad, el $\mathrm{Habib}^{\mathrm{TM}}$ corresponde a un instrumento bipolar que mediante electrodos paralelos insertos en el parénquima hepático aplica energía de radiofrecuencia, produciendo coagulación del tejido hepático central (precoagulación del tejido) para su posterior sección. El TissueLink ${ }^{\mathrm{TM}}$ también ocupa energía por radiofrecuencia, la cual al combinarse con el suero que va rodeando la punta del instrumento, produce coagulación permitiendo la transección y sellado de los vasos. El WaterJet ${ }^{\mathrm{TM}}$ corresponde a un propulsor de agua que va fragmentando el tejido hepático y exponiendo las estructuras vasculares y biliares. Por último se debe mencionar que los vasos mayores y conductos biliares que van emergiendo se controlan con ligaduras, clips o suturas vasculares, mientras que la sección del pedículo hepático en sí y de las venas suprahepáticas cuando son necesarios se lleva a cabo mediante stapler (Figura 4).

\section{b. Hemostasia}

Para lograr una buena hemostasia, al momento de ir realizando la sección, se debe ir inmediatamente controlando todos los sangrados provenientes del parénquima hepático expuesto. Para lograr esto se puede realizar simple compresión con gasa u ocupar instrumentos clásicos como el Hook, que usa energía monopolar. Existen varios otros coaguladores tales como el bipolar y los que permiten coagular en una mayor superficie como son el coagulador de argón y el de microondas. El coagulador de argón se usa junto al monopolar para fulgurar superficies y realizar coagulación superficial del parénquima expuesto. Finalmente, existen una serie de otros productos tales como los sellantes de fibrina, hemostáticos reabsorbibles en base a celulosa y matrices hemostáticas que se combinan con trombina, que permiten asegurar la hemostasia una vez completada la transección.

\section{i. Extracción de la pieza operatoria}

Habitualmente se usa el endo-bag para la extracción, dado la fragilidad del tejido hepático y el tamaño de las piezas resecadas, sobre todo cuando se enfrenta patología maligna, evitando en este caso el contacto de la pieza operatoria con el instrumental y tejidos extrahepáticos. Cuando el procedimiento es mano asistido la extracción se realiza sin problemas a través del puerto usado por la mano del cirujano. Cuando el procedimiento es laparoscópico puro las opciones van desde la ampliación de uno de los puertos a una minilaparotomía, la extracción a través de una laparotomía previa como un McBurney por ejemplo o la realización de una laparotomía de Pfannenstiel (Figura 5).


Figura 4. Transección hepática con disector ultrasónico y hemostasia con bipolar. Los vasos mayores requieren hemostasia con stapler.

Figura 5. Extracción de la pieza operatoria en endo-bag a través de laparotomía de Pfannenstiel. 


\section{j. Drenaje}

El uso del drenaje es variable dependiendo del centro. Algunos no dejan habitualmente, aunque la mayoría sí lo hace luego de hepatectomías mayores o cuando quedan dudas luego de la transección. La recomendación actual es usar un drenaje, sobre todo en hepatectomías mayores o ante dudas de una adecuada identificación de estructuras biliares.

\section{Técnicas específicas}

Los aspectos específicos a considerar variarán según el segmento a resecar y el centro en que nos encontremos. Las hepatectomías mayores más importantes son:

\section{Segmentectomía lateral laparoscópica (segmentos II y III)}

- Paciente en posición de Lloyd-Davies con el cirujano entre las piernas y ambos ayudantes a la izquierda.

- Neumoperitoneo de $\mathrm{CO}_{2}$ con aguja de Veress hasta $15 \mathrm{~mm} \mathrm{Hg}$, definir con precisión la ubicación de los trócares una vez insuflado el abdomen. Uso rutinario de 2 insufladores.

- Instalación de 4 trócares: 1) Supra umbilical de $10 \mathrm{~mm}$ en línea media; 2) Subcostal izquierdo de $5 \mathrm{~mm}$, línea medio clavicular; 3) Hipocondrio derecho de $5 \mathrm{~mm}$ en línea medio clavicular y 4) Hipocondrio izquierdo de $10 \mathrm{~mm}$ en línea axilar anterior.

- Cámara con óptica de $30^{\circ}$ y EIOL.

- Liberar el hígado seccionando el ligamento gastrohepático, coronario y triangular izquierdos.

- Identificar la vena supra hepática izquierda e intentar aislarla, aunque no siempre es posible dado su emergencia conjunta con la vena supra hepática media (Figura 6).

- Sección del puente de parénquima hepático entre los segmentos III y IV.

- Transección del parénquima $1 \mathrm{~cm}$ aproximada- mente a la izquierda del ligamento falciforme.

- Identificación de pedículos portales de los segmentos II y III y sección de ellos con stapler.

- Sección de la vena supra hepática izquierda con stapler.

- Ectomía de la pieza.

\section{Hepatectomía derecha laparoscópica}

- Paciente en posición de Lloyd-Davies con el cirujano entre las piernas y ambos ayudantes a la izquierda.

- Neumoperitoneo de $\mathrm{CO}_{2}$ con aguja de Veress hasta $15 \mathrm{~mm} \mathrm{Hg}$, definir con precisión la ubicación de los trócares una vez insuflado el abdomen. Uso rutinario de 2 insufladores.

- Instalación de 5 trócares: 1) Supra umbilical de $12 \mathrm{~mm}$ en línea media; 2) Subcostal izquierdo de $12 \mathrm{~mm}$, línea medio clavicular; 3) Subxifoideo de $5 \mathrm{~mm}$; 4) Hipocondrio derecho de $12 \mathrm{~mm}$ paralelo a trócar supraumbilical y 5) Subcostal derecho de $5 \mathrm{~mm}$ en línea axilar anterior.

- Cámara con óptica de $30^{\circ}$ y EIOL.

- 3 etapas:

a. Disección portal:

- Disección del pedículo hépato-duodenal por vía póstero-lateral derecha, para evitar daño de estructuras izquierdas contralaterales (Figura 7).

- Disección de la arteria hepática derecha, ligadura con doble clip y sección con tijera.

- Disección de rama derecha de vena porta identificando bifurcación portal. Ligadura con doble Hem-o-Lok® y sección con tijera, previa comprobación de delimitación isquémica del parénquima hepático (Figura 8).

- El conducto biliar derecho no se diseca en esta etapa y se secciona junto a la transección parenquimatosa (Figura 9).

- Se rodea todo el pedículo con cinta para control vascular (maniobra de Pringle), éste

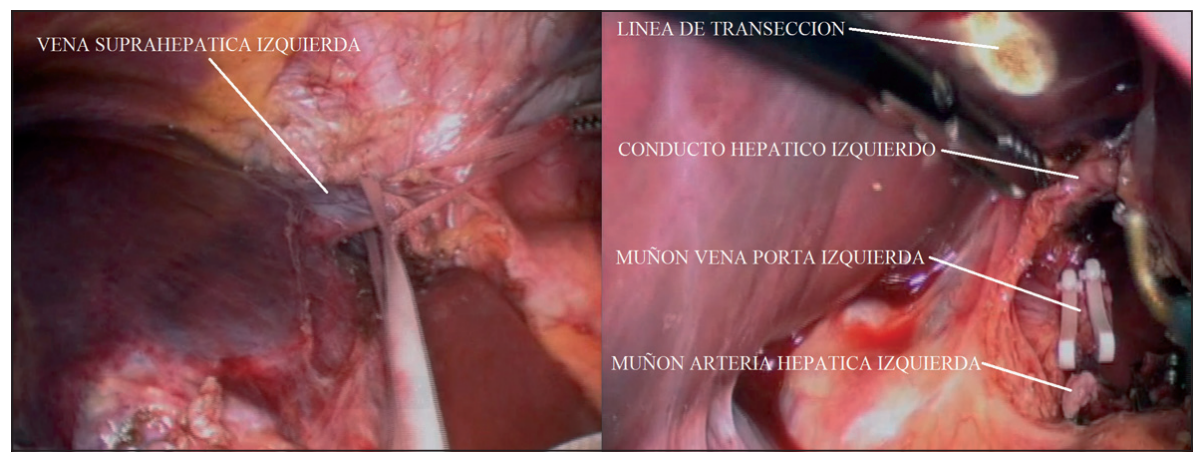

Figura 6. Disección extrahepática e identificación con cinta de la vena suprahepática izquierda. A la derecha se observa la disección del conducto hepático izquierdo. 
puede ser realizado con cinta de seda y tubo de silicón o con el uso de clamps vasculares laparoscópicos.

b. Disección vena cava:

- Levantar todo el parénquima hepático con 2 graspers.

- Confeccionar túnel retrohepático, ligando las venas hepáticas menores con bisturí harmoni$\mathrm{co}^{\mathrm{TM}}$ o clips (Figura 10).

- Una vez divisada la vena supra hepática derecha seccionar con stapler (Figura 11). Alternativamente en casos que no es posible una adecuada disección de esta vena, sobre todo con la dificultad que entrega el ligamento intercavo, puede diferirse su sección al término de la transección parenquimatosa. c. Transección del parénquima:

- Sección a través de la línea de demarcación con bisturí harmonico ${ }^{\mathrm{TM}}$ y stapler para venas de mayor calibre.

- Hemostasia del borde de sección con bipolar.

- Finalmente, sección de los ligamentos hepáticos derechos.

- Remoción de la pieza operatoria con endo-bag.

- Cuando no se logra adecuada exposición se puede convertir a procedimiento mano asistido a través de incisión subcostal der.

\section{Hepatectomía izquierda laparoscópica}

- Paciente en posición de Lloyd-Davies con el cirujano entre las piernas y ambos ayudantes a la izquierda.

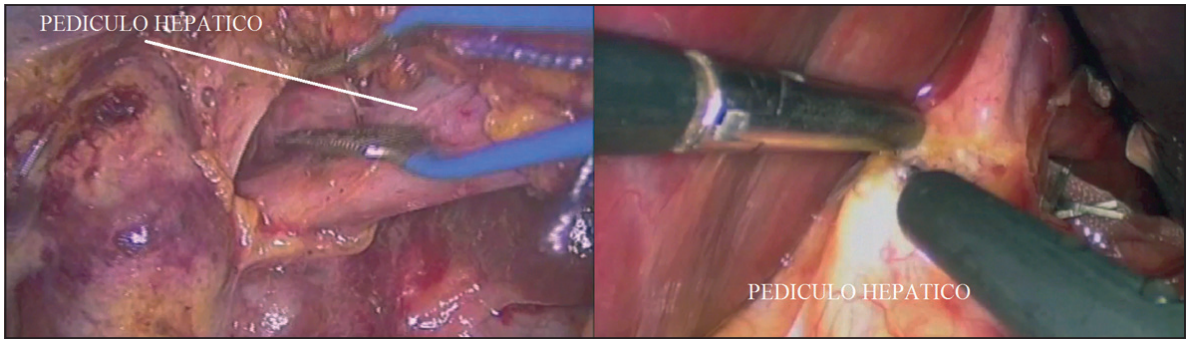

Figura 7. Disección del pedículo hepático derecho e izquierdo, respectivamente.


Figura 8. Disección de la vena porta derecha y sección con tijera.
Figura 9. Disección intrahepática del conducto hepático derecho y sección con stapler. 
- Neumoperitoneo de $\mathrm{CO}_{2}$ con aguja de Veress hasta $15 \mathrm{~mm} \mathrm{Hg}$, definir con precisión la ubicación de los trócares una vez insuflado el abdomen. Uso rutinario de 2 insufladores.

- Instalación de 5 trócares: 1) Supra umbilical de $12 \mathrm{~mm}$ en línea media; 2) Subcostal izquierdo de $12 \mathrm{~mm}$, línea medio clavicular; 3) Subxifoideo de $5 \mathrm{~mm}$; 4) Hipocondrio derecho de $12 \mathrm{~mm}$ paralelo a trócar supraumbilical y 5) Hipocondrio izquierdo de $12 \mathrm{~mm}$ en línea medio clavicular.

- Cámara con óptica de $30^{\circ}$ y EIOL.

- 3 etapas:

a. Liberación del hígado seccionando ligamentos falciforme, coronario y triangular izquierdos (Figura 12).

b. Disección vena supra hepática izquierda:

- Una vez liberado el ligamento triangular y coronario izquierdos, se secciona el ligamento de Arantius hasta identificar límites de vena supra hepática izquierda por encima del segmento caudado.

- Disección de vena cava supra hepática e intentar rodear vena supra hepática izquierda. Puede ser seccionada con stapler vascular o mantener aislada hasta el final de la transección (Figura 6). En caso que esta no se separe adecuadamente de la vena supra hepática media, se puede diferir esta etapa hasta el final de la transección del parénquima (Figura 13).

c. Disección portal:

- Disección del pedículo hépato-duodenal por vía anterior izquierda, para evitar daño de estructuras derechas contralaterales (Figura 7).

- Disección de la arteria hepática izquierda, ligadura con doble clip y sección con tijera (Figura 14).
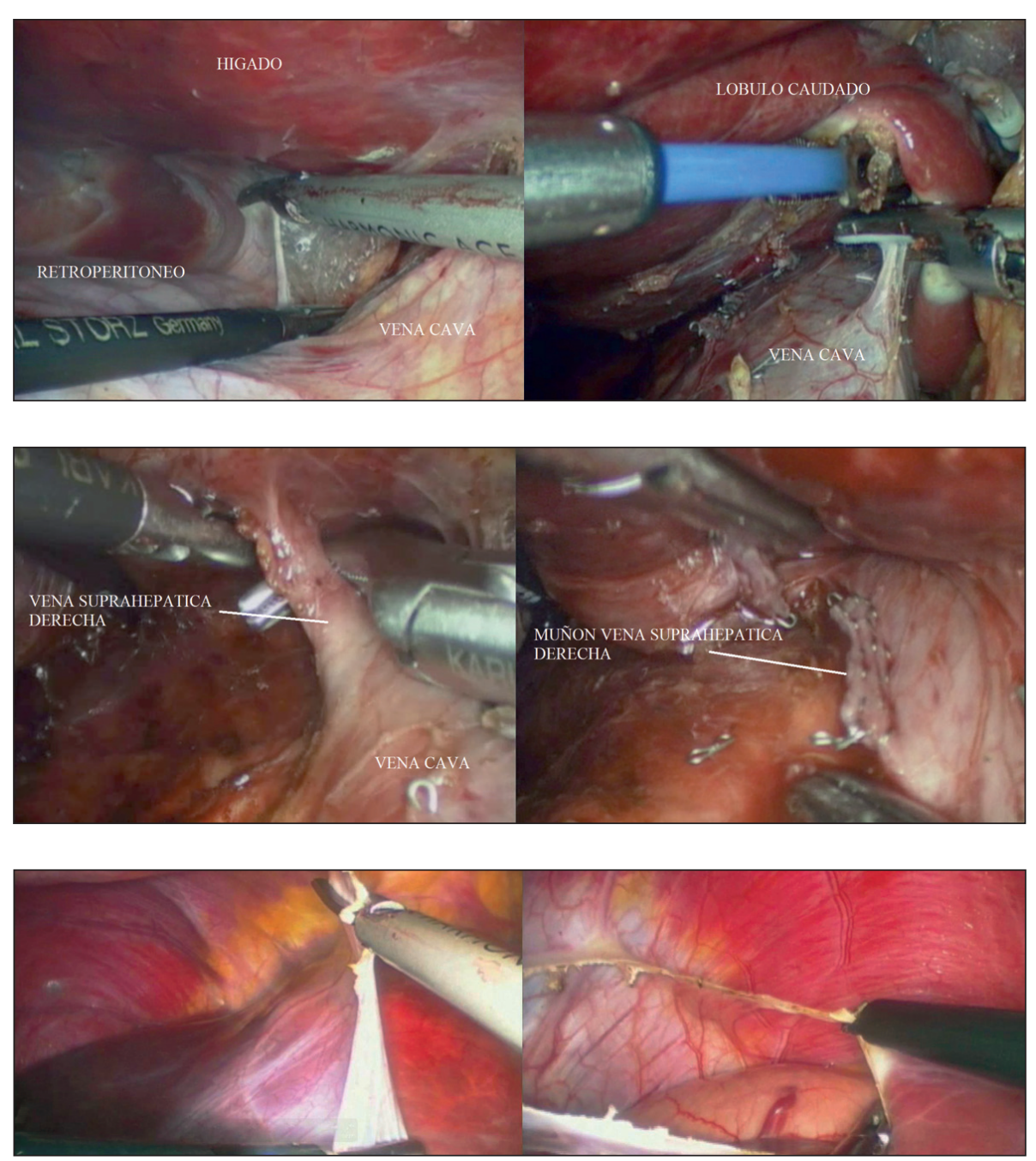

Figura 10. Confección de túnel retrohepático seccionando ramas del lóbulo caudado a la vena cava.

Figura 11. Disección retrohepática de la vena suprahepática derecha y sección con stapler.

Figura 12. Sección del ligamento falciforme. 
- Disección de rama izquierda de vena porta identificando bifurcación portal. Ligadura con doble Hem-o-Lok® y sección con tijera, previa comprobación de delimitación isquémica del parénquima hepático (Figura 15). Alternativamente sección con stapler vascular.

- Identificación de conducto biliar izquierdo pero no se secciona hasta completar la transección hepática vecina al conducto (Figura 6).

- Se rodea todo el pedículo con cinta para control vascular (maniobra de Pringle), este puede ser realizado con cinta de seda y tubo de silicón o con el uso de clamps vasculares laparoscópicos.

\section{d. Transección del parénquima:}

- Sección a través de la línea de demarcación con bisturí harmonico ${ }^{\mathrm{TM}}$ y stapler para venas de mayor calibre.

- Hemostasia del borde de sección con bipolar.

- Sección del conducto hepático izquierdo.

- Sección del parénquima y conducto de Arantius por sobre el segmento caudado.
- Sección de vena supra hepática izquierda (Figura 13).

- Remoción de la pieza operatoria con endo-bag.

\section{Donante vivo laparoscópico}

En la cirugía de donante vivo los pasos son similares a los descritos para la resección de segmentos laterales, pero con los siguientes cambios específicos:

- La movilización del hígado izquierdo debe ser extremadamente cuidadosa, especialmente al seccionar los ligamentos.

- Durante la disección del pedículo izquierdo, el flujo arterial y portal deben ser preservados durante toda la cirugía, incluida la transección del parénquima (Figura 16).

- Usualmente 2 ó 3 ramas de la porta izquierda al caudado deben ser disecadas y seccionadas para una adecuada liberación de esta vena. De esta forma se logra una mayor longitud de la vena porta izquierda para el implante.
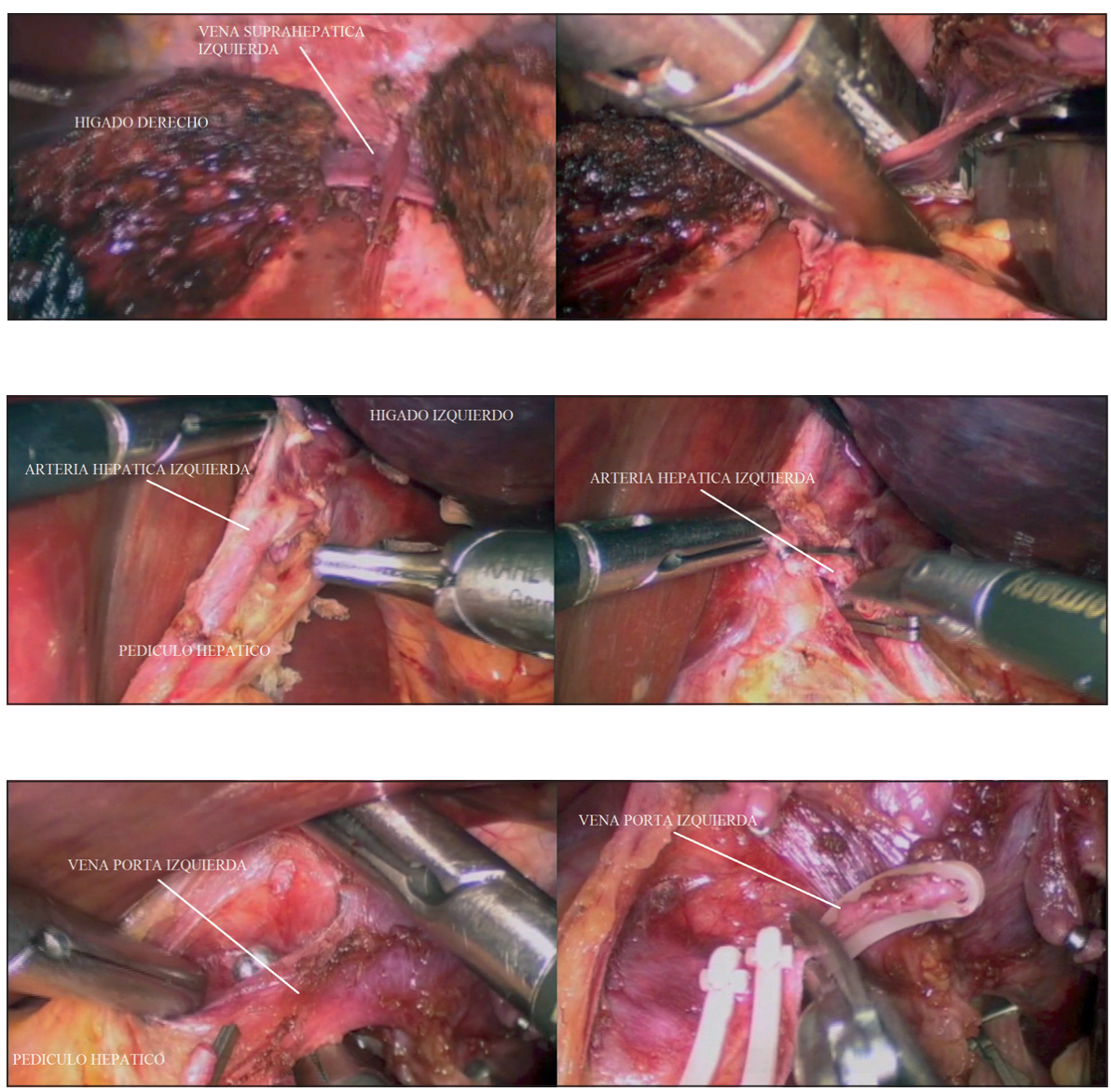

Figura 13. Disección de la vena suprahepática izquierda durante la transección y sección con stapler.

Figura 14. Disección de la arteria hepática izquierda y sección con tijera.
Figura 15. Disección de la vena porta izquierda y sección con tijera. 


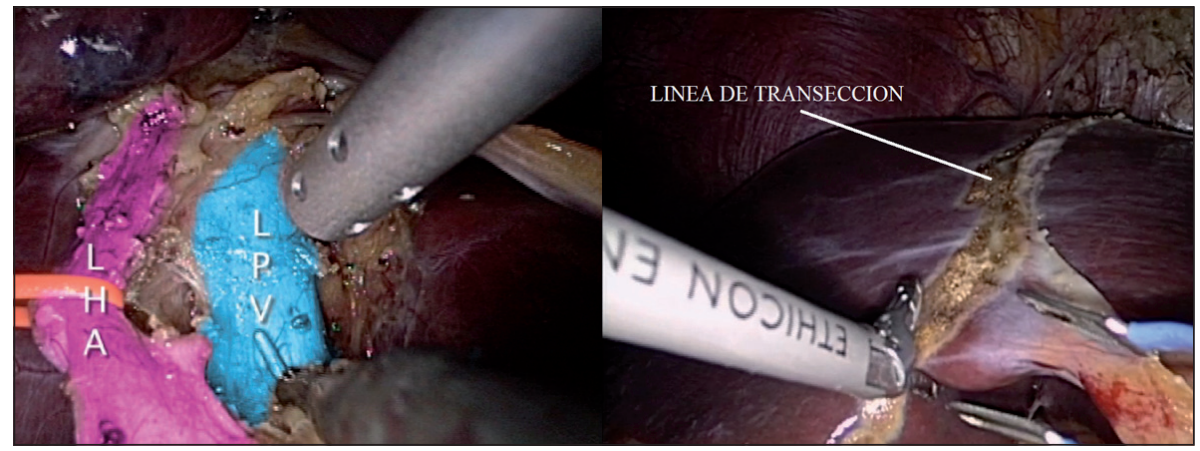

Figura 16. Disección del pedículo izquierdo. En la hepatectomía de donante vivo el flujo arterial y portal debe ser mantenido durante todo el procedimiento, incluyendo la transección hepática.

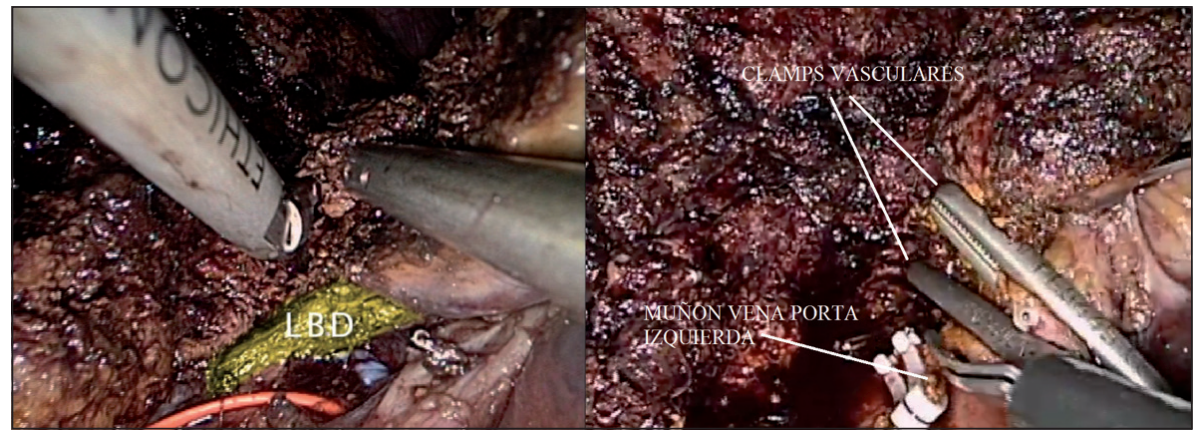

Figura 17. Identificación del conducto hepático izquierdo durante la disección del pedículo. A la derecha se observa la sección de la vena porta izquierda luego de la instalación de dos Hem-o-Lok® y clamps vasculares.

- Extremo cuidado se debe tener al disecar el conducto hepático izquierdo. Este debe ser visualizado pero no seccionado hasta que la transección esté completa y luego de descartar variaciones anatómicas tales como comunicaciones al conducto hepático derecho o confluencia. A través de una colangiografía trancística se puede obtener una mejor definición de la anatomía biliar (Figura 17).

- Una vez finalizada la transección, se instalan clamps vasculares en la arteria hepática y vena porta izquierdas, evitando así el daño endotelial que puede ocurrir con las ligaduras (Figura 17).

- Previo a la sección del pedículo hepático se realiza una laparotomía de Pfannenstiel para la extracción de la pieza operatoria, a fin de minimizar el daño isquémico.

- Finalmente, las estructuras del pedículo son seccionadas, seguidas por la vena supra hepática izquierda (Figura 18).

\section{Referencias}

1. Dagher I, O'Rourke N, Geller DA, Cherqui D, Belli G, Gamblin C et al. Laparoscopic major hepatectomy. An evolution in standard of care. Ann Surg. 2009;250: 856-60.

2. Nguyen KT, Gamblin C and Geller DA. World review of laparoscopic liver resection-2,804 patients. Ann Surg. 2009;250:831-41.

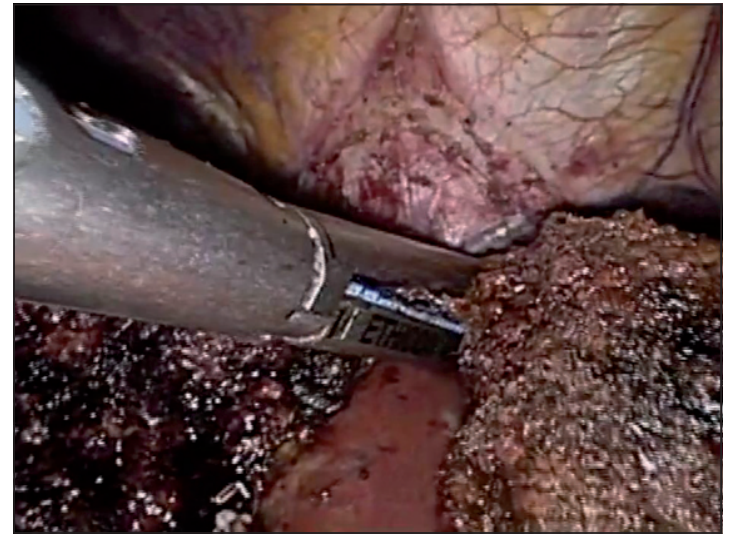

Figura 18. Sección de la vena suprahepática izquierda con stapler una vez completada la transección hepática.

3. Viganò L, Ferrero A, Amisano M, Russolillo N, Capussotti L. Comparison of laparoscopic and open intraoperative ultrasonography for staging liver tumours. $\mathrm{Br} \mathrm{J}$ Surg. 2013;100:535-42.

4. Smyrniotis V, Farantos C, Kostopanagiotou G, Arkadopoulos N. Vascular control during hepatectomy: review of methods and results. World J Surg. 2005;29:138496.

5. Poon RT. Current techniques of liver transection. HPB (Oxford) 2007;9:166-73. 